\title{
Autoconcepto e inteligencia emocional en función de la danza para la expresión lingüístico comunicativa y artística
}

\author{
Lidia Pellicer ${ }^{1}$, Inmaculada Méndez ${ }^{1}$, y Alba Mª́pez ${ }^{2}$ \\ ${ }^{1}$ Universidad de Murcia (España); ${ }^{2}$ ISEN Centro Universitario (España)
}

\begin{abstract}
Diversos estudios han determinado que la ejecución de proyectos que utilicen como nuevas metodologías la danza o la música suponen un bienestar personal y grupal. Así dichas metodologías implican repercusiones para la salud tanto a nivel físico como mental y psíquico. El objetivo del estudio fue analizar si la implicación en un proyecto sobre danza presentaba algún efecto en el autoconcepto y en la empatía de los estudiantes. Los participantes del estudio fueron 31 jóvenes universitarios, con edades comprendidas entre los 18 y los 43 años, pertenecientes a diferentes estudios de Ciencias de la Educación de una universidad del suroeste español. Los instrumentos de evaluación, tanto en el pretest como el postest, fueron el test de autoconcepto AF5 y Test de Empatía Cognitiva y Afectiva (TECA). La prueba t de student determinó diferencias de medias significativas entre el grupo control y el grupo experimental que había participado en el proyecto de danza en el autoconcepto académico, el autoconcepto emocional y el autoconcepto físico. Los datos del estudio permitirán tener en cuenta la implicación de los jóvenes universitarios en proyectos que involucren a la danza debido a los beneficios que presentan.
\end{abstract}

Palabras clave: Comunicación lingüística, autoconcepto, danza, interdisciplinariedad, inteligencia emocional.

Self-concept and emotional intelligence as a function of dance for communicative and artistic linguistic expression. Various studies have determined that the execution of projects that use dance or music as new methodologies entail personal and group well-being. Thus, these methodologies imply repercussions for health both physically, mentally and psychically. The aim of the study was to analyze whether involvement in a dance project had any effect on students' self-concept and empathy. The study participants were 31 young university students, aged between 18 and 43, belonging to different Education Sciences studies at a university in southwestern Spain. The assessment instruments, both in the pretest and the posttest, were the AF5 self-concept test and the Cognitive and Affective Empathy Test (TECA). Student's t-test determined significant mean differences between the control group and the experimental group that had participated in the dance project in academic self-concept, emotional self-concept and physical self-concept. The data of the study will allow taking into account the involvement of young university students in projects that involve dance due to the benefits they present.

Keywords: Linguistic communication, self-concept, dance, interdisciplinarity, emotional intelligence 
Diversos estudios han determinado que la ejecución de proyectos que utilicen como nuevas metodologías la danza o la música suponen beneficios personales y grupales. Así dichas metodologías implican repercusiones para la salud tanto a nivel físico como mental y psíquico (Abilleira, Fernández-Vilallarino, Ramallo, y Prieto, 2017; De Rueda y López, 2013; Murgui, García, García, y García, 2012; Pérez-Aldeguer, 2017). Del mismo modo, se ha constatado que los programas de danza permiten la expresión emocional (De Rueda y López, 2013; Méndez y Pellicer, 2018) así como el desarrollo del autoconcepto (Abilleira et al., 2017). Por lo que se ha de tener en cuenta el papel del autoconcepto (García, Musitu, y Veiga, 2006) y de la empatía en el bienestar personal del individuo (Fernández-Pinto, López-Pérez, y Márquez, 2006).

Los beneficios psicosociales de la danza y el movimiento para el individuo son indiscutibles (Pascual, 2010, p. 324). El arte es una actividad eminentemente social, sea cual sea la expresión del mismo y puesto que forma parte de la vida cotidiana del individuo podemos considerarlo como un lenguaje (Ros, 2004).

Asimismo, la evidencia ha dejado patente el hecho de que los practicantes de danza suelen tener un mayor interés por la práctica de la actividad física (Álvarez et al., 2015; Goñi e Infante, 2010) lo que a su vez presenta importantes beneficios en el autoconcepto, sobre todo el autoconcepto físico, de las personas practicantes a diferencia de los no practicantes (Álvarez, Cuevas, Lara, y González, 2015; Fernández, Feu, y Suárez, 2020).

Para ello, en nuestro estudio el proceso de enseñanza y aprendizaje se focalizó en la aplicación de conocimientos convirtiéndose, de esta forma, los procedimientos, las habilidades, las estrategias, las actitudes y los valores en objeto de la educación. Todo ello teniendo en cuenta las competencias de las titulaciones presentes en las Guías Docentes de las asignaturas implicadas en el estudio, pertenecientes a una universidad del sureste español, así como las transversales genéricas (instrumentales, personales y sistémicas) en relación con los perfiles profesionales desarrollados en el proyecto, según el modelo del proyecto tuning analizado en el Libro Blanco de Magisterio (ANECA, 2005, p. 81-86). Concretamente, y coincidiendo con las tres competencias más valoradas según la muestra de ANECA, "la capacidad de comunicación oral y escrita en lengua materna, la capacidad de organización y planificación y la capacidad para el reconocimiento de la diversidad y la multiculturalidad" (ANECA, 2005, p. 85). Además, se tuvieron en cuenta las tres siguientes en mejor estimación: "habilidades en las relaciones interpersonales, creatividad y trabajo en equipo" (ANECA, 2005, p. 85). La puesta en juego de dichas habilidades se activó mediante la resolución de situaciones diversas de forma eficaz en contextos determinados, de modo que se trabajó por competencias, transformando el proceso de docencia y de aprendizaje de pasivo a activo. Dentro de este enfoque activo de enseñanza/aprendizaje (Cassany, Sanjuan, y Sanz, 2007; Martín, 2009; Prado, 2004; Zabala y Arnau, 2007), se integra la danza como manifestación artística y, por tanto, comunicativa (Pagola, 2016). Este proceso de comunicación se desprende de las posibilidades motrices que nos brinda nuestro cuerpo. Ya que, ajustando los movimientos a un tiempo concreto y espacio, acompañados de una música dada se genera un proceso comunicativo que da como resultado un significado o mensaje (De Rueda y López, 2013). 
En nuestro proyecto el aprendizaje de las danzas se llevó a cabo mediante la metodología flipped-classroom o clase invertida (Santiago y Bergman, 2018). La concreción de este proyecto se realizó desde una vertiente interdisciplinar conjugando las habilidades lingüístico-comunicativas (hablar, escuchar, leer y escribir) con las artísticas y el desarrollo del autoconcepto a través de un proyecto de Inteligencia creativa vertebrado por la danza en el que se imbricaron las disciplinas de Comunicación lingüística, Música y Psicología; y considerando a los estudiantes como gestores de su propio proceso de aprendizaje.

Por lo tanto, el objetivo del estudio fue analizar si la implicación en un proyecto sobre danza presentaba algún efecto en el autoconcepto y en la empatía de los jóvenes universitarios.

\section{MÉTODO}

\section{Participantes}

Los participantes del estudio fueron 31 jóvenes universitarios (93.5\% mujeres y el $6.5 \%$ varones) de una universidad del suroeste español con edades comprendidas entre los 18 y los 43 años $(M=22.81, D T=6.35)$, pertenecientes a estudios de Ciencias de la Educación (13 estudiantes formaron parte del grupo experimental y 18 estudiantes formaron parte del grupo control). El 10\% del alumnado había nacido fuera de España.

\section{Instrumentos}

Se administraron dos instrumentos de evaluación tanto en el grupo control como en el grupo experimental. A continuación, se describen brevemente.

En primer lugar, para evaluar el autoconcepto el instrumento utilizado fue el test de autoconcepto AF5, Autoconcepto Forma 5 (García y Musitu, 1999). Este cuestionario evalúa cinco dimensiones del autoconcepto: el autoconcepto social (hace referencia a las percepciones que tiene el sujeto acerca de su desempeño en las relaciones interpersonales), el autoconcepto familiar (hace alusión a la percepción del sujeto su nivel de implicación, grado de participación e integración a nivel familiar), el autoconcepto académico-laboral (hace referencia a la percepción del sujeto sobre el desempeño de su rol como estudiante o trabajador), el autoconcepto emocional (hace alusión a la percepción general del individuo sobre su estado emocional así como sus respuestas emocionales a situaciones específicas) y el autoconcepto físico (se refiere a la percepción del individuo sobre su aspecto y condición física). El alfa de Cronbach para el total de la escala es de 0.82. Ejemplo de ítems: "Hago amigos fácilmente".

En segundo lugar, para evaluar la capacidad de empatía se seleccionó el Test de Empatía Cognitiva y Afectiva (TECA) elaborado por López-Pérez, Fernández-Pinto, y Abad (2008). Consta de 33 ítems que, siguiendo el modelo integrador de Davis (1980) e incluyendo la distinción de Eisenberg y Strayer (1987) sobre la adopción de perspectiva empática, evalúa tanto aspectos cognitivos como afectivos de la empatía. Entre los procesos cognitivos podemos encontrar la adopción de perspectivas (capacidad para ponerse en el lugar de otra persona) y la comprensión emocional (la capacidad para reconocer y comprender los estados emocionales de los otros). Y entre los procesos emocionales podemos encontrar: el estrés empático (la 
capacidad de compartir emociones negativas de otra persona) o la alegría empática (la capacidad de compartir emociones positivas de otras personas). El alfa de Cronbach para el total de la escala es de 0.86. Ejemplo de ítems: "Me resulta difícil darme cuenta de las intenciones de los que me rodean".

El proyecto consistió en varias actividades que se desarrollaron a lo largo del cuatrimestre durante una sesión semanal para componer la coreografía final.

\section{Procedimiento}

Previamente se solicitó la autorización necesaria en la Universidad en la cual se iba a desarrollar el proyecto de innovación docente. A continuación, se seleccionaron los estudiantes que formaban parte del grupo experimental (participación en las actuaciones previstas en el proyecto, el cual se componía de una coreografía que se estructuraba en varias actividades llevadas a cabo a lo largo de un cuatrimestre incluyendo una representación final) y por otro lado, el grupo control (estudiantes matriculados en las mismas materias pero que no participaban en el proyecto de danza). Durante todo el proceso se aseguró la confidencialidad, la voluntariedad y el anonimato de los datos.

\section{Análisis de datos}

Atendiendo a las características del estudio y el contexto en el que se aplicó, se trataba de un diseño cuasiexperimental con grupo de control no equivalente debido a que no se asignaron a los participantes de forma aleatoria (Ato y Vallejo, 2015).

Se utilizaron técnicas descriptivas (frecuencias, porcentajes, media y desviación típica) así como análisis de medias a través de la prueba $t$ de student para muestras independientes utilizando grupo (control/ danza) como variable categórica dicotómica y como variables dependientes continuas las siguientes: autoconcepto académico-laboral; autoconcepto social; autoconcepto emocional, autoconcepto familiar y autoconcepto físico; adopción de perspectivas, comprensión emocional, estrés empático y alegría empática. Se tuvo en cuenta el análisis del tamaño del efecto de Cohen (d). Todos los análisis se llevaron a cabo a través del paquete estadístico SPSS -Statistical Package for the Social Sciences- (versión 24.0).

\section{RESULTADOS}

En lo que respecta al autoconcepto, la prueba t de Student mostró diferencias de medias significativas, asumiendo varianzas homogéneas, entre los estudiantes que habían participado en el proyecto de danza (grupo experimental) frente a los que no $t_{(29)}=2.256 ; p=.032$ con tamaño del efecto alto $(d=0.87)$ en autoconcepto académico. Así, la media de los estudiantes que habían realizado el proyecto de danza fue superior a la media de los estudiantes del grupo control (Véase en la Tabla 1).

Asimismo, el autoconcepto emocional mostraba diferencias de medias significativas, asumiendo varianzas homogéneas, en función del grupo de danza o del grupo control $t_{(29)}=2.120 ; p=.043$ con tamaño del efecto medio- alto $(d=0.79)$. La media de los 
estudiantes que habían realizado danza resultó ser más alta que la media de los estudiantes del grupo control.

Por último, el autoconcepto físico mostró diferencias de medias significativas, asumiendo varianzas homogéneas, en función del grupo de danza o del grupo control $t_{(29)}=2.564 ; p=.016$ con tamaño del efecto medio- alto $(d=0.79)$. Siendo la media de los estudiantes que habían realizado danza más alta que la media de los estudiantes del grupo control.

No se encontraron diferencias en el autoconcepto social ni en el autoconcepto familiar.

Tabla 1. Medias y desviaciones típicas en las dimensiones del autoconcepto en función del grupo (danza/control)

\begin{tabular}{lccc}
\hline Dimensiones del Autoconcepto & & Danza & $\begin{array}{c}\text { Grupo } \\
\text { Control }\end{array}$ \\
\hline \multirow{2}{*}{ Autoconcepto académico } & $M$ & 7.28 & 6.4 \\
& $D T$ & $(0.49)$ & $(1.33)$ \\
\hline \multirow{2}{*}{ Autoconcepto social } & $M$ & 7.65 & 7.72 \\
& $D T$ & $(1.40)$ & $(1.34)$ \\
\hline \multirow{2}{*}{ Autoconcepto emocional } & $M$ & 5.25 & 3.84 \\
& $D T$ & $(1.60)$ & $(1.97)$ \\
\hline \multirow{2}{*}{ Autoconcepto familiar } & $M$ & 8.48 & 8.6 \\
\multirow{2}{*}{ Autoconcepto físico } & $D T$ & $(1.97)$ & $(1.16)$ \\
& $M$ & 6.99 & 5.5 \\
& $D T$ & $(1.67)$ & $(1.61)$ \\
\hline
\end{tabular}

En lo que respecta a los aspectos afectivos y cognitivos de la empatía, no se encontraron diferencias significativas en la prueba $t$ de Student entre las subescalas en función del grupo (danza/control) a pesar de que algunas medias resultaron ser ligeramente superiores en el grupo experimental (Véase la tabla 2).

Tabla 2. Medias y desviaciones típicas en los aspectos cognitivos y afectivos de la empatía en función del grupo (danza/control)

\begin{tabular}{lccc}
\hline \multicolumn{3}{l}{ Aspectos cognitivos y afectivos de la empatía } & \multicolumn{2}{l}{ Danza } & Grupo Control \\
\hline \multirow{2}{*}{ Adopción de perspectivas } & $M$ & 28.15 & 27.44 \\
& $D T$ & $(2.70)$ & $(2.33)$ \\
\hline \multirow{2}{*}{ Comprensión empática } & $M$ & 30.23 & 30.61 \\
& $D T$ & $(2.92)$ & $(3.84)$ \\
\hline \multirow{2}{*}{ Estrés empático } & $M$ & 19.85 & 22.11 \\
& $D T$ & $(2.99)$ & $(3.86)$ \\
\hline \multirow{2}{*}{ Alegría empática } & $M$ & 31.62 & 30.28 \\
& $D T$ & $(1.98)$ & $(3.29)$ \\
\hline
\end{tabular}

\section{DISCUSIÓN Y CONCLUSIONES}

Los resultados del estudio evidenciaron que los estudiantes del grupo experimental presentaron diferencias de medias más altas en el autoconcepto académico, emocional y físico en comparación con el grupo control. Por lo que se ha encontrado que los practicantes de danza tienen un mayor autoconcepto (Abilleira et al., 2017; Murgui et al., 2012). Esto está en la línea con estudios anteriores que evidencian que los practicantes de danza suelen tener un mayor interés por la actividad física, debido sobre todo al tiempo que dedican a dicha actividad y por 
las experiencias que comparten. Esto implica que la práctica de la actividad física está asociada a un mayor autoconcepto físico, lo que a su vez se ha relacionado con una mayor satisfacción con la vida y por ende un mayor bienestar psicológico (Álvarez et al., 2015; Goñi e Infante, 2010). La práctica de la danza se ha asociado a la felicidad y satisfacción lo que a su vez influye en la autoestima del sujeto (Abilleira et al., 2017) y a la expresión emocional (De Rueda y López, 2013; Méndez y Pellicer, 2018). En estudios anteriores se evidenció que la danza promovía la empatía entre los participantes (Méndez y Pellicer, 2018) pero en nuestro estudio, a pesar de que existían ligeras diferencias entre los aspectos afectivos y cognitivos de la empatía entre el grupo control y el grupo experimental, no resultaron ser estadísticamente significativas.

Los datos del estudio permitirán tener en cuenta la implicación de los jóvenes universitarios en proyectos que involucren a la danza debido a los beneficios que presentan sobre el autoconcepto académico, el autoconcepto emocional, así como el autoconcepto físico debido a la importancia de la actividad física en los jóvenes universitarios (Álvarez et al., 2015; Fernández et al., 2020). Ello supone que este tipo de metodologías mejoran el aprendizaje, así como la imagen del propio estudiante (Abilleira et al., 2017). Por lo tanto, se considera necesario que los docentes universitarios se involucren desde las diferentes áreas en el desarrollo de este tipo de proyectos (desde el área lingüística, artística, psicológica...) debido al bienestar personal y grupal. Sin duda, el ejercicio corpóreo que conlleva la danza contribuye a un mejor equilibrio psicológico (Pascual, 2010).

Como limitaciones del estudio cabe tener en cuenta en primer lugar, el hecho de que los datos sean autoinformados asimismo la deseabilidad social podría haber distorsionado los resultados. En segundo lugar, para evaluar la capacidad empática se seleccionó el Test de Empatía Cognitiva y Afectiva (TECA) elaborado por López-Pérez et al. (2008) que sigue el modelo integrador de Davis (1980) incluyendo tanto aspectos cognitivos como afectivos de la empatía por lo que en futuras investigaciones puede ser de interés utilizar otros instrumentos que permitan evaluar otras dimensiones de la empatía (Fernández-Pinto et al., 2006).

Entre las prospectivas se plantea la necesidad de llevar a cabo estudios longitudinales desde etapas educativas anteriores para analizar si se mantiene estable con la edad, asimismo se hace preciso hacer comparaciones con otros practicantes de otras actividades físicas o deportivas, incluso diferenciar entre las diferentes especialidades de danza (Murgui et al., 2012), tener en cuenta las estrategias de afrontamiento así como las habilidades sociales (Morales, 2017), la influencia de la ansiedad (De Castro, 2019), entre otros aspectos.

Finalmente, cabe destacar que son escasas las investigaciones que aborden el autoconcepto en el ámbito universitario por lo que es necesario aumentar dichas investigaciones (Abilleira, et al., 2017), así como la implantación de metodologías que involucren las artes escénicas en la educación superior (Pérez-Aldeguer, 2017).

Para concluir, queremos destacar el hecho de que la danza y la música han estado presentes a lo largo de la vida del individuo. Suponen formas de expresar con nuestro cuerpo lo que en ocasiones es difícil expresar con palabras. Así, con la música o la danza se permite conectar con el cuerpo y de ese modo expresar nuestras emociones. Por lo que la danza permite ser un cauce a nivel emocional (De Rueda y López, 2013). 


\section{REFERENCIAS}

Abilleira González, M., Fernández-Vilallarino, M.A., Ramallo, S., y Prieto, J. (2017). Influencia de la Danza en el autoconcepto del alumnado de Educación Primaria. Análisis comparativo con otras actividades físicas. Sportis. Scientific Journal of School Sport, Physical Education and Psychomotricity, 3(3), 554-568. Doi:10.17979/sportis.2017.3.3.2244

Álvarez, L., Cuevas, R., Lara, A., y González, J. (2015). Diferencias del autoconcepto físico en practicantes y no practicantes de actividad física en estudiantes universitarios. Cuadernos de Psicología del Deporte, 15(2), 27-34.

ANECA (2005). Libro Blanco. Título de Grado en Magisterio. Madrid: ANECA.

Ato, M., y Vallejo, G. (2015). Diseños de Investigación en Psicología. Madrid, España: Ediciones Pirámide (Grupo Anaya).

Blanco, H., Benavides, E.V., Tristán, J.L., y Mayorga-Vega, D. (2017). Actividad física, imagen corporal y autoconcepto personal en jóvenes universitarias mexicanas. Revista de Psicología del Deporte, 26(2), 25-33.

Cassany, D., Sanjuan, M.L., y Sanz, G. (2007). Enseñar Lengua. Barcelona: Graó.

Davis, M. H. (1980). A multidimensional approach to individual differences in empathy. Catalog of Selected Documents in Psychology, 10(85), 1-17.

De Castro, P. (2019). Análisis de la relación entre ansiedad rasgo y experiencia profesional en la danza. European Journal of Child Development, Education and Psychopathology, 7(1), 15-22.

De Rueda, B., y López, C.E. (2013). Música y programa de danza creativa como herramienta de expresión de emociones. Retos. Nuevas tendencias en Educación Física, Deporte y Recreación, 24, 141-148.

Eisenberg, N., y Strayer, J. (1987). Empathy and its development. Cambridge University Press.

Fernández, M., Feu, S., y Suárez, M. (2020). Autoconcepto físico en función de variables sociodemográficas y su relación con la actividad física. Cultura, Ciencia y Deporte, 15(44), 189-199. doi:10.12800/ccd.v15i44.1461

Fernández-Pinto, I., López-Pérez, B., y Márquez, M. (2006). Empatía: Medidas, teorías y aplicaciones en revisión. Anales de psicología, 24(2), 284-298.

García, F., y Musitu, G. (1999). AF-5 Autoconcepto Forma 5. Madrid, España: TEA.

García, J.F., Musitu, G., y Veiga, F. (2006). Autoconcepto en adultos de España y Portugal. Psicothema, $18,551-556$.

Goñi, E., e Infante, G. (2010). Actividad físico-deportiva, autoconcepto físico y satisfacción con la vida. European Journal of Education and Psychology, 3(2), 199-208.

López-Pérez, B., Fernández-Pinto, I., y Abad, F.J. (2008). TECA. Test de Empatía Cognitiva y Afectiva. Madrid: Tea Ediciones, S.A.

Martín, R. (2009). Manual de didáctica de la lengua y la literatura. Madrid: Editorial Síntesis.

Méndez, I., y Pellicer, L. (2018). Promoción de la empatía a través de la danza. En P. Miralles Martínez y C. Guerrero Romera (Ed.). Metodologías docentes innovadoras en la enseñanza universitaria (pp.50-57). Murcia: Universidad de Murcia, Editum.

Morales, F. (2017). Relaciones entre afrontamiento del estrés cotidiano, autoconcepto, habilidades sociales e inteligencia emocional. European Journal of Education and Psychology, 10(2), 41-48.doi:10.30552/ejep.v10i2.121

Murgui, S., García, C., García, Á., y García, F. (2012). Autoconcepto en jóvenes practicantes de danza y no practicantes: Análisis factorial confirmatorio de la escala AF5. Revista de Psicología del Deporte, 21(2), 263-269.

Pagola, H. (2016). La danza, ¿comprensión y comunicación a través del cuerpo en movimiento? Brocar, 40, 269-293.doi:10.18172/brocar.3251

Pascual, M. (2010). Didáctica de la música. Madrid: Pearson.

Pérez-Aldeguer, S. (2017). Las artes escénicas como metodología educativa en la educación superior. Foro de Educación, 15(22), 1-7.

Prado, J. (2004). Didáctica de la lengua y la literatura para educar en el siglo XXI. Madrid: La Muralla. 
Ros, N. (2004). El lenguaje artístico, la educación y la creación. Revista Iberoamericana de Educación, 35(1),1-8. doi:10.35362/rie3512901

Santiago, R., y Bergman, J. (2018). Aprender al revés: Flipped learning 3.0 y metodologías activas en el aula. Barcelona: Paidós.

Zabala, A., y Arnau, L. (2007). 11 Ideas clave. Cómo aprender y enseñar competencias. Barcelona: Graó.

Recibido: 12 de agosto de 2020

Recepción Modificaciones: 22 de septiembre de 2020

Aceptado: 24 de septiembre de 2020 